\title{
Obstrucción de la acción climática en la extrema derecha española: La enmienda de Vox a la Ley de Cambio Climático y su representación en prensa
}

\author{
Climate action obstruction in the Spanish far right: The Vox's amendment \\ to the Climate Change Law and its press representation
}

\author{
Jose A. Moreno \\ Universitat Pompeu Fabra | Roc Boronat, 138, 08018 Barcelona | España | \\ https://orcid.org/0000-0003-3781-5644 | joseantonio.moreno@upf.edu \\ Gina Thornton \\ Independent researcher | Roc Boronat, 138, 08018 Barcelona | España | \\ https://orcid.org/0000-0002-8333-908X | gina.thorn8@gmail.com
}

Fechas | Recepción: 30/09/2021 | Aceptación: 14/12/2021

\section{Resumen}

Aunque vivimos un momento crucial en el que la ciencia del clima nos insta a tomar medidas contundentes para mitigar el cambio climático, existe un contramovimiento que obstaculiza la adopción de políticas climáticas. En Europa, hay partidos de extrema derecha que se han alineado con el contramovimiento climático. Esta investigación pretende examinar el discurso sobre el cambio climático del partido de extrema derecha español Vox, así como la recepción en la prensa de las ideas del partido sobre el tema. Este trabajo analiza la enmienda a la totalidad del Proyecto de Ley de Cambio Climático y Transición Energética (121/00019) presentada por Vox el 17 de junio de 2020 en el Parlamento español. El estudio consiste en un examen de los encuadres contrarios a la acción climática siguiendo la tipología de Almiron et al. (2020). A esta investigación le sigue un análisis crítico del discurso de la cobertura de esta enmienda en la prensa española. Este análisis de prensa permite

\section{Abstract}

While we live in a crucial time when climate science urges us to take strong action to mitigate climate change, there is a countermovement obstructing the adoption of climate policies. In Europe, there are far right parties that have aligned their positions with the climate countermovement. This research seeks to examine the climate change discourse of the Spanish far-right party Vox, as well as the press reception of the party's ideas on the subject. This paper analyses the amendment to the whole Law Project on Climate Change and Energetic Transition (121/00019) presented by Vox on June 17, 2020, at the Spanish Parliament. The study consists of an examination of the climate action contrarian framings following the typology of Almiron et al. (2020). This enquiry is followed by a critical discourse analysis of the coverage of this amendment in the Spanish press. This press analysis provides an insight into whether journalists received Vox's proposal critically and 
conocer si los periodistas recibieron la propuesta de Vox de forma crítica y se alinearon con el consenso científico, si informaron de forma neutral, o si apoyaron sus ideas. Los resultados incluyen una reproducción de los manidos argumentos negacionistas del cambio climático difundidos por grupos de interés contrarios a la acción climática en Europa y los Estados Unidos. Este trabajo también identifica una reacción crítica de la prensa española a la enmienda de Vox, a pesar de la existencia de algunos textos que informan de forma neutral sobre la propuesta.

Palabras clave: cambio climático, crisis climática, obstruccionismo, negacionismo, contramovimiento climático. aligned themselves with the scientific consensus, whether they reported it in a neutral way, or whether they supported its ideas. The results include a replication of hackneyed climate change denialist arguments as disseminated by European and North American climate action contrarian stakeholders. This paper also identifies a critical reaction from the Spanish press to this Vox amendment, despite the existence of some texts that report neutrally on the proposal.

Keywords: climate change, climate crisis, obstructionism, denialism, climate countermovement.

\section{INTRODUCCIÓN}

Cada vez tenemos más pruebas de que la actividad humana ya ha tenido un impacto en el clima, produciendo un calentamiento global a un ritmo sin precedentes en los últimos dos milenios (IPCC, 2021). Este cambio climático antropogénico está afectando a la situación meteorológica y, por tanto, produciendo fenómenos climáticos extremos en todo el mundo (IPCC, 2021). Esta circunstancia se agravará si el incremento de las temperaturas respecto a la era preindustrial supera los $1,5 \circ \mathrm{C}$, como ocurrirá si no se frena la actual trayectoria de las emisiones de efecto invernadero (IPCC, 2021). Pero el cambio climático es un tema politizado, y las políticas climáticas no están a la altura de la emergencia descrita por la ciencia del clima. En este escenario politizado, hay actores que tratan de impulsar la adopción de políticas climáticas eficaces (o movimiento climático), y hay otros que obstruyen esos esfuerzos (contramovimiento climático). En este escenario en el que la ciencia del clima ha aportado abundantes pruebas sobre la base física del cambio climático inducido por el ser humano, las humanidades y las ciencias sociales deben tomar la iniciativa. Por qué la humanidad no está respondiendo adecuadamente a tal amenaza es una cuestión compleja que requiere de todas las disciplinas de las ciencias sociales, para examinar los mecanismos que llevaron a la inacción.

En el contexto español no existe un fuerte contramovimiento climático como el que existe en los EEUU o el Reino Unido. Sin embargo, hay actores relevantes que intentan influir en las políticas climáticas para que no se adopten o, si lo hacen, que sea en una versión suavizada. Uno de estos actores es el partido español de extrema derecha Vox. A pesar de la existencia de investigaciones sobre el discurso y el desarrollo político de este partido, existe un desconocimiento sobre la cuestión específica de su postura ante el cambio climático. El objetivo de este trabajo es llenar este vacío examinando los encuadres contrarios a la acción climática mostrados por el partido en la enmienda a la totalidad del Proyecto de Ley de Cambio Climático y Transición Energética (121/00019), presentada por Vox el 17 de junio de 2020 en el Parlamento español. Este análisis se complementa con un análisis crítico del discurso de la representación periodística de esta enmienda de Vox en la prensa española. 


\section{REVISIÓN DE LITERATURA}

El contramovimiento climático, a menudo llamado "maquinaria negacionista" (Piltz, 2008), está formado por empresas, coaliciones de opositores, empresas de relaciones públicas, organizaciones de lobby, fundaciones conservadoras, think tanks, medios de comunicación, políticos, periodistas y blogueros (CSSN, 2021). Como resume la Climate Social Science Network (2021), esta estructura de oposición tiene tres objetivos principales: influir en la agenda pública para asegurar que la opinión pública no apoye la acción climática; moldear los medios de comunicación para poner en duda las acciones para abordar el cambio climático, e influir en el proceso de elaboración de políticas para obstruir la adopción de políticas climáticas. El abanico de actividades que desarrolla este contramovimiento es amplio y va desde la creación y difusión de conocimientos hasta el lobby más directo.

A pesar de la evidencia científica sobre la crisis climática, el terreno político está polarizado sobre cómo abordarla. Se sabe que en EE.UU. los conservadores han tendido a mostrar más a menudo posturas contrarias a la lucha contra el cambio climático, difundiendo lo que McCright y Dunlap (2010) Ilamaron "anti-reflexividad". Esta consiste en la oposición al movimiento ecologista y a la ciencia, promoviendo la no toma de decisiones y la inacción ante la crisis climática. Esto ha producido un debate político polarizado sobre cuestiones climáticas (Fisher et al., 2013) en el que los conservadores han tratado de defender el statu quo, promovido por la financiación corporativa destinada a este fin (Farrell, 2016). En general, existe una correlación entre el apoyo a los partidos populistas de derecha y el escepticismo climático o la oposición a los impuestos climáticos (Lockwood, 2018). En Europa, con el auge de los partidos populistas de extrema derecha existe un creciente interés académico por el posicionamiento climático de estos grupos políticos, especialmente desde el ámbito de la comunicación (p. ej., Forchtner, 2019; Forchtner et al., 2018; Hultman et al., 2019; Jylhä y Hellmer, 2020; Vowles y Hultman, 2021). Los partidos populistas de derechas en Europa suelen presentar posturas contrarias a la acción climática, tal y como informa el think tank Adelphi (Schaller y Carius, 2019). De los veintiún partidos de derecha populista en Europa, siete se mostraron escépticos sobre el consenso climático o lo negaron, once no presentaron una postura sobre el cambio climático y solo dos apoyaron el consenso climático (Schaller y Carius, 2019). En España, la oposición más dura a la adopción de políticas climáticas ha estado representada por el partido populista de extrema derecha Vox, que se ha posicionado firmemente en contra de la existencia de la emergencia climática y de la adopción de medidas para afrontarla (p. ej., González, 2021; Robaina, 2019).

El Congreso de los Diputados era uno de los últimos parlamentos europeos sin representación de la derecha radical, junto con el vecino Portugal, hasta que Vox, un partido de extrema derecha, católico, nacionalista y populista, se convirtió en el tercero más votado en las elecciones españolas de 2019 (Gould, 2019). Vox tiene un fuerte discurso nacionalista que sitúa a los "españoles primero", combinado con un bajo nivel de nativismo, que es sustituido por una importante fijación y motivación en torno al proceso separatista catalán y la defensa de España y las tradiciones españolas (Turnbull-Dugarte, 2019). Vox ha despertado el interés de la investigación en el campo de las ciencias políticas en cuanto a sus orígenes, auge e ideología (p. ej., Ferreira, 2019; Rama et al., 2021). Asimismo, en el campo de la comunicación Vox ha sido examinado por su retórica, en plataformas como las redes sociales (Aladro Vico y Requeijo Rey, 2020), o por su uso de emociones como el miedo en la construcción de su 
discurso (Cárdenas-Rica y Lozano González, 2020). Sin embargo, a pesar de la relevancia de las ideas de Vox sobre el cambio climático, faltan investigaciones sobre el discurso climático de este partido o sobre cómo es recibido por los medios de comunicación.

En este contexto en el que un contramovimiento climático busca socavar las políticas climáticas, e incluso algunos grupos políticos siguen ese camino, el periodismo es una pieza clave para trasladar la ciencia y la acción climática de forma efectiva a la sociedad (Boykoff, 2019; Boykoff y Farrell, 2020). Pero, aunque el negacionismo del cambio climático no tiene una presencia considerable en la prensa española (Martín-Sosa, 2021), esta sí que está fallando al dar al cambio climático el rigor y la atención que requiere (p. ej., Fernández-Reyes, 2014; Parratt Fernández et al., 2020) e incluso está invisibilizando a importantes sectores emisores de gases de efecto invernadero como el de la agricultura animal (Moreno y Almiron, 2021). También es importante señalar que la cobertura periodística sobre el cambio climático está sometida a la presión estructural de aquellos actores involucrados en empresas contaminantes o con interés en obstruir la acción climática (Mancinas-Chávez, 2013). Un ejemplo de ello es el lavado verde que recibieron las empresas energéticas patrocinadoras de la COP25 ChileMadrid en la prensa española por su inversión en estrategias de publicidad y relaciones públicas (Moreno y Ruiz-Alba, 2021). El carácter multidisciplinar de este campo de estudio y su relevancia dada la crisis climática nos anima a contribuir a esta línea de investigación con estudios empíricos como el que aquí se propone.

\section{METOdOLOGÍA}

Los objetivos de investigación de este trabajo son dos. Primero, comprobar si la enmienda a la totalidad del Proyecto de Ley de Cambio Climático y Transición Energética (121/00019), presentada por Vox el 17 de junio de 2020 en el Parlamento español, presenta los argumentos contrarios a la acción climática más comunes difundidos por los think tanks europeos y estadounidenses contrarios a la acción climática. $Y$, en segundo lugar, descubrir cómo la prensa cubrió la presentación de dicha enmienda.

Para responder al primer objetivo, se ha realizado un análisis de encuadres (frames). Un encuadre es una determinada interpretación de la realidad, una definición, un diagnóstico, un juicio o una solución determinada para un problema (Entman, 1993). Para identificar los argumentos contrarios al clima utilizados en la enmienda de Vox se ha utilizado la lista de contramarcos sobre el cambio climático definida por Almiron et al. (2020) que se muestra en la Tabla 1.

Tabla 1

Encuadres contrarios a la acción climática

A. Afirmaciones científicas generales:

A1. Cuestionamiento de la legitimidad del IPCC (implícita o explícitamente)

A2. Cuestionamiento del consenso y legitimidad de la ciencia climática (no del IPCC)

A3. Cuestionamiento de la difusión científica (por políticos, medios u otros)

B. Afirmaciones científicas específicas:

B4. No está ocurriendo (el cambio climático o el calentamiento global) 
B5. Está ocurriendo, pero no sabemos cómo de serio es o si no es serio

B6. Está ocurriendo, pero es bueno/no es malo (el calentamiento global o aspectos particulares de este)

B7. Está ocurriendo, pero no somos nosotros o no somos solo nosotros (otros elementos son causas de este o la causa principal)

B8. Está ocurriendo, pero tenemos otros problemas importantes

B9. Está ocurriendo, pero cualquier política será peor que el calentamiento

\section{Afirmaciones no científicas:}

C10. Crítica de los defensores no científicos, mensajes y políticas con argumentos no científicos

C11. El texto incluye una posición económica neoliberal o neoconservadora (apoyando el crecimiento económico como solución, la autorregulación de los mercados, la mínima intervención gubernamental, la no imposición de la contaminación, etc.)

C12. El texto incluye una mención a la población humana como problema

C13. El texto incluye una mención a las dietas de proteínas animales o a la agricultura animal como problema

C14. El texto confía en la tecnología como solución al cambio climático o a sus consecuencias

Fuente: Almiron et al. (2020).

Vox presentó una enmienda a la totalidad al Proyecto de Ley de Cambio Climático y Transición Energética (121/00019) el 17 de junio de 2020, cuando este texto legislativo estaba abierto a la discusión de los partidos. La enmienda fue rechazada, y la ley fue aprobada el 20 de mayo de 2021, convirtiéndose en la primera ley sobre cambio climático aprobada en el parlamento español. Vox votó en contra por considerarla "dogmática".

Para abordar el segundo objetivo, recopilamos un corpus de textos de la prensa española. Se utilizó la plataforma Factiva introduciendo las palabras clave "Vox AND Ley de cambio climático" para un rango de fechas desde el 1 de junio de 2020, hasta el 31 de julio de 2020 (la enmienda fue rechazada el 14 de julio). Esta búsqueda produjo 149 resultados, de los cuales, tras eliminar los duplicados y los textos no válidos, 67 fueron aptos para el análisis. Para analizar estos textos, aplicamos un enfoque de análisis crítico del discurso (ACD). El ACD se puede utilizar para analizar los textos de las noticias con el fin de exponer las estrategias que parecen normales o neutrales en la superficie, pero que en realidad pueden ser ideológicas y tratar de dar forma a la representación de los acontecimientos y las personas con fines particulares (Machin y Mayr, 2012). EI ACD puede considerarse como una metodología comprometida con el cambio político y social, ya que su objetivo es revelar las ideologías y el discurso subyacentes a través del lenguaje (Fairclough y Wodak, 1997). Cuando se aplica una metodología que sigue el ACD, "las categorías y las herramientas no se fijan de una vez por todas. Deben elaborarse para cada análisis según el problema específico que se investigue" (Wodak y Meyer, 2009, p. 111). En este caso, utilizamos las siguientes categorías para el análisis:

- Categoría 1, que aborda la forma en que el discurso mediático describe la acción de Vox.

- Categoría 2, que aborda la cuestión de la representación de los actores sociales. 
- Categoría 3, que abarca la equivalencia y la diferencia entre la construcción del "grupo interno" y el "grupo externo".

Esta combinación de encuadre para el texto y de análisis crítico del discurso para la cobertura de la enmienda por parte de la prensa nos permite comprender qué argumentos están presentes en el documento, y cómo reaccionó la prensa española ante estos argumentos.

\section{RESULTADOS}

\subsection{Análisis de encuadres}

La enmienda de Vox a la ley de cambio climático presenta 11 de los 14 marcos contrarios a la acción climática utilizados por Almiron et al. (2020). La Tabla 2 muestra los extractos de la enmienda que encajan en cada encuadre. Esta categorización deja claro que la enmienda de Vox está muy alineada con los discursos obstruccionistas de la acción climática difundidos por el contramovimiento climático.

Tabla 2

Análisis de encuadres de la enmienda a la totalidad del Proyecto de Ley de Cambio Climático y Transición Energética (121/00019) presentada por Vox el 17 de junio de 2020 en el Parlamento español

\begin{tabular}{|c|c|}
\hline Encuadre & Extracto \\
\hline $\begin{array}{l}\text { A1. Cuestionamiento de } \\
\text { la legitimidad del IPCC } \\
\text { (implícita o } \\
\text { explícitamente) }\end{array}$ & $\begin{array}{l}\text { «Las previsiones alarmistas del Intergovernmental Panel on Climate Change } \\
\text { (en adelante IPCC) se ven desmentidas una y otra vez por la realidad)» (p. 4). }\end{array}$ \\
\hline $\begin{array}{l}\text { A2. Cuestionamiento } \\
\text { del consenso y } \\
\text { legitimidad de la ciencia } \\
\text { climática (no del IPCC) }\end{array}$ & $\begin{array}{l}\text { «El alarmismo climático está haciendo las veces de una nueva religión. Se } \\
\text { agrupan en un solo paquete indisociable -para el cual se exige un } \\
\text { asentimiento sin fisuras, so pena de ser anatemizado como "negacionista"- } \\
\text { proposiciones lógicamente independientes (que la temperatura está } \\
\text { subiendo; que la subida se debe a los gases de invernadero emitidos por el } \\
\text { hombre; que la subida se va a acelerar en este siglo; que esa subida será muy } \\
\text { perjudicial para la humanidad; que vendrá acompañada de todo tipo de } \\
\text { catástrofes -huracanes, sequías, etc.- agrupadas en el cajón de sastre del } \\
\text { "cambio climático")» (p. 3). } \\
\text { «VoX deja la ciencia a los científicos, y carece de una posición propia sobre } \\
\text { cuestiones climatológicas. Considera, sin embargo, que el debate no está } \\
\text { cerrado, y que es irracional asumir como artículo de fe el paquete climático- } \\
\text { catastrofista en su totalidad. [...] los científicos no son concluyentes al } \\
\text { apreciar una correlación clara entre el ritmo de incremento térmico y la } \\
\text { acción humana» (p. } 3 \text { ). }\end{array}$ \\
\hline $\begin{array}{l}\text { A3. Cuestionamiento de } \\
\text { la difusión científica } \\
\text { (por políticos, medios u } \\
\text { otros) }\end{array}$ & $\begin{array}{l}\text { «[... "cambio" puede ser cualquier cosa: cualquier fenómeno (la subida de } \\
\text { temperaturas, pero también su estancamiento, como ocurrió durante “la } \\
\text { Pausa" de 1998-2014, o la de } 1945-75 » \text { (p. 3). }\end{array}$ \\
\hline
\end{tabular}




\begin{tabular}{|c|c|}
\hline $\begin{array}{l}\text { B5. Está ocurriendo, } \\
\text { pero no sabemos cómo } \\
\text { de serio es o si no es } \\
\text { serio }\end{array}$ & $\begin{array}{l}\text { «Se ha pronosticado repetidamente el inminente deshielo de los casquetes } \\
\text { polares, pero la banquisa ártica era en septiembre de } 2019 \text { más gruesa que } \\
\text { en } 2007 \text { según el National Snow \& Ice Data Center de EE.UU., y un estudio de } \\
\text { la NASA publicado en } 2015 \text { en el Journal of Glaciology certificaba que la masa } \\
\text { de hielo antártico, en lugar de disminuir, está aumentando» (p. 4). } \\
\text { «Asegurar que una temperatura dos o tres grados más alta llevará a la } \\
\text { humanidad al desastre a finales del siglo XXI, denota un grado de lucidez } \\
\text { comparable al de los futurólogos que, hacia 1890, pronosticaban que en } \\
\text { treinta años Londres y París yacerían bajo una montaña de estiércol, dado el } \\
\text { crecimiento exponencial del número de coches de caballos» (p. 6). }\end{array}$ \\
\hline $\begin{array}{l}\text { B6. Está ocurriendo, } \\
\text { pero es bueno/no es } \\
\text { malo (el calentamiento } \\
\text { global o aspectos } \\
\text { particulares de este) }\end{array}$ & $\begin{array}{l}\text { «Algunos expertos reivindican el } \mathrm{CO} 2 \text { como elemento fundamental de la vida } \\
\text { en la Tierra, al ser necesario para la fotosíntesis. El CO2 solo es responsable } \\
\text { de un } 15 \% \text { del efecto invernadero (sin efecto invernadero, la temperatura del } \\
\text { planeta sería } 30 \text { grados más baja, y la vida no habría sido posible). El aumento } \\
\text { de } \mathrm{CO} 2 \text { en la atmósfera ha generado efectos positivos que el catastrofismo } \\
\text { climático oculta cuidadosamente» (p. 5). } \\
\text { «El profesor Richard Tol, de la Universidad de Sussex, ha elaborado un } \\
\text { modelo que computa los efectos favorables y desfavorables de la subida de } \\
\text { temperatura: concluye que habrá beneficio neto (ahorro en calefacción, } \\
\text { aumento de cosechas, etc.) hasta un aumento de } 2,2 \text { grados respecto a la } \\
\text { temperatura global de 2009; por encima de ese umbral, los costes superarían } \\
\text { a las ventajas» (p. 5). }\end{array}$ \\
\hline $\begin{array}{l}\text { B8. Está ocurriendo, } \\
\text { pero tenemos otros } \\
\text { problemas importantes }\end{array}$ & $\begin{array}{l}\text { «La OCDE sitúa ya a España a la cabeza del desplome económico mundial; } \\
\text { registramos la mayor destrucción de empleo de Europa y el deterioro del } \\
\text { tejido productivo retrotrae el número de empresas a principios de siglo. La } \\
\text { "emergencia climática" tiene que ser compatible con la auténtica emergencia } \\
\text { económica y la social que enfrentan los españoles. Por consideraciones } \\
\text { elementales de supervivencia nacional, el PLCCTE debe ser devuelto al } \\
\text { Gobierno» (p. 12). }\end{array}$ \\
\hline $\begin{array}{l}\text { B9. Está ocurriendo, } \\
\text { pero cualquier política } \\
\text { será peor que el } \\
\text { calentamiento }\end{array}$ & $\begin{array}{l}\text { «España debe seguir comprometida con el medio ambiente, pero no a } \\
\text { cualquier coste» (p. 1). }\end{array}$ \\
\hline $\begin{array}{l}\text { C10. Crítica de los } \\
\text { defensores no } \\
\text { científicos, mensajes y } \\
\text { políticas con } \\
\text { argumentos no } \\
\text { científicos }\end{array}$ & $\begin{array}{l}\text { «La izquierda, cuya sensibilidad medioambiental es muy reciente, necesita } \\
\text { pretextos para justificar la intervención coactiva del Estado en la economía y } \\
\text { la sociedad: unas veces es 'la pobreza' (que suele multiplicarse como } \\
\text { resultado de las políticas socialistas); otras, la 'opresión de género', o la } \\
\text { 'discriminación racial'. Como todos ellos son fenómenos superados -o } \\
\text { residuales- en la España contemporánea, se ha buscado en la 'emergencia } \\
\text { climática' una nueva excusa para el dirigismo» (p. 2). } \\
\text { "Al rechazar simultáneamente los combustibles fósiles y la energía nuclear, el } \\
\text { "paquete energía y clima” (PLCCTE + PNIEC) rompe con la racionalidad } \\
\text { científica y el realismo económico, condenando a España a la penuria } \\
\text { energética y el empobrecimiento» (p. 11). }\end{array}$ \\
\hline
\end{tabular}




\begin{tabular}{|c|c|}
\hline $\begin{array}{l}\text { C11. El texto incluye una } \\
\text { posición económica } \\
\text { neoliberal o } \\
\text { neoconservadora } \\
\text { (apoyando el } \\
\text { crecimiento económico } \\
\text { como solución, la } \\
\text { autorregulación de los } \\
\text { mercados, la mínima } \\
\text { intervención } \\
\text { gubernamental, la no } \\
\text { imposición de la } \\
\text { contaminación, etc.) }\end{array}$ & $\begin{array}{l}\text { «La búsqueda de esos objetivos requerirá un intervencionismo exhaustivo del } \\
\text { Estado en la vida económica y un incremento brutal del gasto público» (p. 2). } \\
\text { «En Vox consideramos, por el contrario, que este planteamiento puede tener } \\
\text { efectos adversos para la inversión, el consumo, el empleo y la competitividad } \\
\text { de la economía española» (p. 2). } \\
\text { «Las emisiones españolas de CO2 representan un 0'7\% del total mundial. Si } \\
\text { después de esquilmar a los españoles con impuestos verdes y condenar a la } \\
\text { quiebra o la deslocalización a las industrias dependientes de combustibles } \\
\text { fósiles, se alcanzaran los objetivos marcados por el PLCCTE (reducción del } \\
23 \% \text { en las emisiones), nuestra incidencia positiva en el volumen mundial de } \\
\text { emisiones sería de un 0'17\%». (p. 7) } \\
\text { «Las diversas fuentes de energía han de poder competir libremente entre sí, y } \\
\text { debe permitirse a las empresas optar por las más rentables. [...] Si las } \\
\text { renovables llegan a ser realmente rentables, se impondrán por sí mismas, de } \\
\text { la misma forma que las bombillas eléctricas desplazaron a los quinqués y los } \\
\text { automóviles a los coches de caballos» (p. 7). }\end{array}$ \\
\hline $\begin{array}{l}\text { C12. El texto incluye una } \\
\text { mención a la población } \\
\text { humana como } \\
\text { problema }\end{array}$ & $\begin{array}{l}\text { «En ridículos similares incurrieron las predicciones neomaltusianas de Paul } \\
\text { Ehrlich (The Population Bomb, 1968) o el Club de Roma (Informe "Los límites } \\
\text { del crecimiento", 1972), que auguraban un rápido agotamiento de recursos } \\
\text { esenciales debido a la superpoblación. Todos ellos olvidaron que el más } \\
\text { importante de los recursos es, como teorizara Julian Simon, la inteligencia } \\
\text { humana, que permite hacer cada vez más con menos» (p. 6). }\end{array}$ \\
\hline $\begin{array}{l}\text { C14. El texto confía en } \\
\text { la tecnología como } \\
\text { solución al cambio } \\
\text { climático o a sus } \\
\text { consecuencias }\end{array}$ & $\begin{array}{l}\text { «En } 2080 \text {, si se mantiene el ritmo de crecimiento económico de las últimas } \\
\text { décadas, seremos tres veces más ricos que ahora, y la tecnología será capaz } \\
\text { de proezas que ahora no podemos ni soñar» (p. 6). } \\
\text { «El PLCCTE, en su falta de equilibrio por erradicar los combustibles fósiles, } \\
\text { desatiende otros horizontes tecnológicos que permitirían reducir CO2 sin } \\
\text { perjudicar la competitividad empresarial. Por ejemplo, las nuevas técnicas } \\
\text { Carbon Capture, Use and Storage (CCUS) de captación de CO2» (p. 9). } \\
\text { "[...] la energía nuclear es una energía limpia. No emite CO2, y por eso ha sido } \\
\text { defendida por grandes referentes del movimiento ecologista, como James } \\
\text { Lovelock (el padre de la "hipótesis Gaia"); James Hansen, Ken Caldeira, Tom } \\
\text { Wigley y Kerry Emanuel, profetas del clima-catastrofismo» (p. 10). }\end{array}$ \\
\hline
\end{tabular}

Fuente: elaboración propia.

\subsection{Análisis crítico del discurso}

A través del ACD, examinaremos las tres categorías mencionadas, así como el uso del discurso nacionalista, etnocéntrico y tradicionalista en general.

\subsubsection{Categoría 1. Representación del discurso político y de la ideología}

Esta categoría de análisis del discurso político y la ideología se inspira en el trabajo de Fairclough (2003). Examinaremos la agencia (quién hace qué a quién) y la acción (qué se hace), prestando especial atención a los participantes (does y done-tos), al proceso (grupos verbales) y a las circunstancias (grupos adverbiales, frases preposicionales). La Tabla 3 muestra este análisis. 
Tabla 3

Análisis de la representación del discurso político y de la ideología (Categoría 1)

\begin{tabular}{|l|l|}
\hline \multicolumn{1}{|c|}{ Categorías } & \multicolumn{1}{c|}{ Extracto } \\
\hline $\begin{array}{l}\text { Partido de } \\
\text { extrema derecha } \\
\text { (participante) }\end{array}$ & $\begin{array}{l}\text { «"El clima es cambiante y ha evolucionado durante siglos", ha asegurado la } \\
\text { diputada del partido de ultraderecha Mireia Borrás» (EI Periódico de Catalunya, } \\
14 / 7 / 2020) .\end{array}$ \\
\hline $\begin{array}{l}\text { Subrayado } \\
\text { (proceso) }\end{array}$ & $\begin{array}{l}\text { "La representante del PSOE, Helena Caballero Gutiérrez, subrayó que Vox ni } \\
\text { siquiera se ha tomado la molestia de redactar un texto alternativo. Los } \\
\text { planteamientos de la ultraderecha, dijo Caballero, "atentan contra la racionalidad } \\
\text { científica"» (Sur Online, 14/7/2020) }\end{array}$ \\
$\begin{array}{l}\text { Extrema derecha } \\
\text { (participante) }\end{array}$ & $\begin{array}{l}\text { «"iA ver si nos aclaramos!", ridiculizó esa postura Juantxo Lopez de Uralde, } \\
\text { diputado de Unidas Podemos» (La Vanguardia, 14/7/2020) }\end{array}$ \\
\hline $\begin{array}{l}\text { Ridiculización } \\
\text { (proceso) }\end{array}$
\end{tabular}

Fuente: elaboración propia.

\subsubsection{Categoría 2. Representación de los actores sociales}

Es imposible representar a los actores políticos, o a las personas en general, de forma neutral, y la forma en que caracterizamos a alguien puede tener fines psicológicos, sociales y políticos (Reisigl y Wodak, 2001). El objetivo de esta categoría es establecer cómo los medios de comunicación representan a los actores políticos de Vox y sus círculos circundantes (votantes, simpatizantes, etc.). La Tabla 4 recoge los principales actores sociales de estos textos.

Tabla 4

Análisis de los actores sociales representados (Categoría 2)

\begin{tabular}{|c|c|}
\hline Actor social representado & Extracto \\
\hline $\begin{array}{l}\text { Mireia Borràs, portavoz de } \\
\text { Vox ( } 16 \text { menciones) }\end{array}$ & $\begin{array}{l}\text { "La diputada de Vox Mireia Borrás Pabón, encargada de presentar esta } \\
\text { enmienda a la totalidad, ha asegurado durante su intervención que el } \\
\text { proyecto supone un “intervencionismo exhaustivo del Estado en la vida } \\
\text { económica y un incremento brutal del gasto público" » (EFE/Diario de } \\
\text { Sevilla,14/7/2020) }\end{array}$ \\
\hline $\begin{array}{l}\text { Vox (como partido/ partido } \\
\text { de extrema derecha/ } \\
\text { partido de Santiago } \\
\text { Abascal) ( } 81 \text { menciones) }\end{array}$ & $\begin{array}{l}\text { «El partido que preside Santiago Abascal, también denuncia la } \\
\text { "hostilidad" declarada del Ejecutivo en esta ley a los combustibles fósiles» } \\
\text { (Europa Press, 17/6/2020). }\end{array}$ \\
\hline $\begin{array}{l}\text { Partidarios de Vox ( } 4 \\
\text { menciones) }\end{array}$ & $\begin{array}{l}\text { «Su respuesta contrasta con la de los votantes de Vox, entre los cuales } \\
\text { solo uno de cada cinco se declara contrario a la exploración de } \\
\text { hidrocarburos» (El Mundo, 8/6/2020). }\end{array}$ \\
\hline $\begin{array}{l}\text { Teresa Ribera } \\
\text { (Vicepresidenta del } \\
\text { Gobierno, PSOE, en el } \\
\text { cargo) (19 menciones) }\end{array}$ & $\begin{array}{l}\text { «En su intervención, Ribera ha rechazado los principales argumentos que } \\
\text { Vox utiliza en su enmienda a la totalidad para rechazar el texto del } \\
\text { Gobierno» (Europa Press, 14/7/2020). }\end{array}$ \\
\hline
\end{tabular}




\begin{tabular}{|l|l|}
\hline $\begin{array}{l}\text { Juantxo López de Uralde } \\
\text { (diputado, Unidas } \\
\text { Podemos) (7 menciones) }\end{array}$ & $\begin{array}{l}\text { "Juantxo López de Uralde, se ha preguntado qué "patriotismo" es que el } \\
\text { defiende Vox cuando niega el "daño" que el cambio climático está } \\
\text { haciendo a su territorio» (Europa Press, 14/7/2020). }\end{array}$ \\
\hline $\begin{array}{l}\text { Ciudadanía Española, } \\
\text { mayormente granjeros, } \\
\text { cazadores y ganaderos (7 } \\
\text { menciones) }\end{array}$ & $\begin{array}{l}\text { "Vox no compra estos dogmas que "culpan a los españoles", } \\
\text { especialmente a los ganaderos, agricultores y cazadores» (Europa Press, } \\
14 / 7 / 2020) .\end{array}$ \\
\hline
\end{tabular}

Fuente: elaboración propia.

Aquí podemos ver un claro caso de funcionalización: los ciudadanos españoles más afectados son definidos en función de su trabajo y, además del partido, los principales actores están relacionados por su papel en el proceso. La más interesante es, sin embargo, es la última categoría y el uso de la visión tradicional de la agricultura española ("ciudadanos, agricultores, cazadores, ganaderos") y el uso indirecto de esta categoría en el discurso. Esto encaja perfectamente en el marco discursivo de la extrema derecha de utilizar ciertos argumentos, como esta funcionalización, relativos a los valores nacionales y tradicionales, para enfocar su discurso y acciones como un acto de preservación de la cultura y tradición etno-nacional.

\subsubsection{Categoría 3. Equivalencia y diferencia}

El objetivo de esta categoría es comprender la construcción del "grupo interno" (in-group) y del "grupo externo" (out-group), y la diferencia entre las representaciones positivas y negativas de cada uno. Para ello hemos utilizado una adaptación de las estrategias de equivalencia y diferencia de Wodak (2009).

Tabla 5

Análisis de la equivalencia y la diferencia a través de la referencialidad/nominación (Categoría 3)

\begin{tabular}{|c|c|}
\hline Equivalencia y diferencia & Extracto \\
\hline $\begin{array}{l}\text { In-group (según Vox): } \\
\text { Patriotas, agricultores, ganaderos } \\
\text { Vs. } \\
\text { Out-group, los otros (según Vox): PSOE / Gobierno } \\
\text { de España / La izquierda }\end{array}$ & $\begin{array}{l}\text { «Mireia Borrás Pavón, portavoz de Vox, aseguró } \\
\text { que el departamento que dirige Teresa Ribera } \\
\text { pretende "culpabilizar a agricultores y ganaderos" } \\
\text { del cambio climático» (La Verdad, 15/7/2020). }\end{array}$ \\
\hline $\begin{array}{l}\text { In-group (según Vox): } \\
\text { La ciudadanía española, que está atravesando una } \\
\text { crisis socioeconómica } \\
\text { Vs. } \\
\text { Out-group, los otros (según Vox): } \\
\text { La élite interesada en el cambio climático }\end{array}$ & $\begin{array}{l}\text { "Vox (...) al considerar que se trata de un texto que } \\
\text { no es compatible con "la auténtica emergencia } \\
\text { económica y social a la que se enfrentan los } \\
\text { españoles" y, por tanto, pone en peligro la } \\
\text { "supervivencia nacional”» (Europa Press, } \\
\text { 17/6/2020). }\end{array}$ \\
\hline
\end{tabular}

Fuente: elaboración propia.

\subsubsection{Análisis de los discursos nacionalistas, tradicionalistas y euroescépticos}

Para terminar, hemos abordado la aparición en la prensa de los discursos de Vox anclados en el nacionalismo, el tradicionalismo y el euroescepticismo al hablar de la ley de cambio climático. La Tabla 6 resume algunos ejemplos. 
Obstrucción de la acción climática en la extrema derecha española: La enmienda de Vox a la Ley de Cambio Climático

Tabla 6

Análisis de la equivalencia y la diferencia a través de la referencialidad/nominación (Categoría 3)

Extractos

"efectos adversos en la inversión, el consumo, el empleo y en la competitividad económica española"» (Europa Press, 17/6/2020).

"pone en peligro la "supervivencia nacional" " (Europa Press, 17/6/2020).

"Vox cree que estos objetivos están diseñados para (...) "transformar radicalmente" (...) "hasta el modo de vida de los españoles"» (Europa Press, 18/6/2020).

" "Son los lobbys energéticos los que están marcando la agenda europea"» (Europa Press, 4/6/2020).

"Vox ha criticado la "liberticida" ley de Cambio Climático del Gobierno que, a su juicio, "quiere cambiar la economía y la vida de los españoles" " (Europa Press, 14/7/2020).

Fuente: elaboración propia.

Estos resultados son la representación de la legitimación de Vox de su postura a través del uso de la terminología nacionalista, prueba de cómo los partidos de extrema derecha buscan transmitir valores tradicionalistas, etnonacionalistas e incluso económico-proteccionistas (menos comunes) a través de sus argumentos en cualquier ámbito, incluido el cambio climático.

\subsubsection{La enmienda de Vox a la ley de cambio climático en la prensa española: una síntesis}

El retrato que hace la prensa española de la enmienda a la ley de cambio climático presentada por Vox es predominantemente crítico. El análisis de los participantes en el discurso periodístico (actores políticos citados) y los procesos que se reflejan, muestran que la enmienda de Vox representa una posición minoritaria y no se alinea con el consenso científico. Así, la propuesta del partido de extrema derecha es ridiculizada y mostrada como carente de soporte argumental.

Por su parte, el análisis de los actores sociales implicados refleja una sobrerrepresentación de Vox. A pesar de la matización del discurso de Vox y de la inclusión de actores que rechazan la enmienda propuesta por el partido, hay carencias en la inclusión de otras voces (de ámbitos como la ciencia, el activismo o la sociedad civil) que contrarresten los discursos de extrema derecha emitidos por el partido y recogidos por la prensa.

El discurso de Vox delimita un in-group formado por el partido y sus simpatizantes, dejando a los políticos progresistas en el gobierno en un out-group. Esta representación y dicotomización contribuye a la polarización de un tema tan sensible como el cambio climático, sobre el que es necesario alcanzar consensos y acuerdos que faciliten la adopción de políticas climáticas.

Por último, este análisis muestra cómo los ideales de Vox (nacionalismo, tradicionalismo e incluso algo de euroescepticismo) se entrelazan con su discurso climático, apuntalando su posición reaccionaria hacia la adopción de políticas de mitigación del cambio climático.

\section{DISCUSIÓN Y CONCLUSIONES}

Esta investigación cubre un vacío de conocimiento sobre el discurso de Vox en materia de cambio climático. En concreto, se analiza el discurso de Vox con respecto a la ley española de cambio climático aprobada en 2021 a través de una enmienda realizada por el partido en 
2020. Esta enmienda está claramente alineada con los discursos contrarios a la acción climática más comunes en Estados Unidos y Europa, ya que incluye los principales argumentos negacionistas y obstruccionistas más utilizados por las organizaciones en esta línea. Estos discursos emplean mecanismos retóricos que ya han sido analizados y desmontados en numerosas ocasiones (p. ej.: Cook et al., 2018; McKie, 2019). Esta investigación muestra una similitud entre los encuadres utilizados por Vox en su enmienda y los emitidos por los principales think tanks europeos contrarios a la acción climática estudiados por Almiron et al. (2020). De hecho, cabe destacar aquí que Vox cita en su enmienda a Richard Tol, uno de los autores colaboradores de la Global Warming Policy Foundation, un popular think tank inglés conocido por su oposición a la acción climática (Black, 2018). Además, el ex director del Instituto Juan de Mariana (otro de los think tanks obstruccionistas estudiados por Almiron et al. 2020), Eduardo Fernández Luiña, es subdirector del think tank de Vox, la Fundación Disenso.

En cuanto a la recepción por parte de la prensa de la enmienda de Vox a la ley de cambio climático, la cobertura es eminentemente crítica. Sin embargo, se han detectado carencias en cuanto a la inclusión de voces que contrarresten el discurso de Vox en la prensa, marcado por la polarización y el uso de elementos nacionalistas para defender su posición contraria a la acción climática. En este sentido, sería útil que la prensa proporcionara al público más herramientas para identificar el contramovimiento climático y evitar lagunas que pongan en duda el consenso científico sobre el cambio climático (van der Linden et al., 2017).

El discurso de Vox se alinea así también con las posiciones opuestas a la acción climática de otros partidos europeos de extrema derecha que describen Schaller y Carius (2019). A este respecto, Forchtner y Lubarda (2021) indican que los partidos de extrema derecha están enfocando sus posicionamientos hacia la obstrucción de las políticas climáticas y no tanto hacia la negación de la ciencia climática, es decir, son más obstruccionistas que negacionistas. En el caso de la enmienda a la ley de cambio climático presentada por Vox, hay encuadres de negación del consenso científico sobre el cambio climático, de rechazo de las proyecciones científicas y de crítica a la ciencia del clima, sin aparecer el encuadre de negación literal de la existencia del cambio climático. Pero, pese a la importancia de este tipo de argumentos engañosos que aducen una supuesta falta de consenso científico, destacan los encuadres contrarios a las políticas climáticas en base a diferentes argumentos ideológicos y económicos, lo que concuerda con lo expuesto por Forchtner y Lubarda (2021). A este respecto, Forchtner y Lubarda (2021) alertan de que los partidos de extrema derecha pueden utilizar los argumentos contrarios a la acción climática "para generalizar su 'otredad' de, por ejemplo, los inmigrantes, los liberales y los izquierdistas, 'la élite' y las organizaciones internacionales", una situación que otros autores como Lockwood (2018) también han señalado. El análisis crítico del discurso realizado en este artículo corrobora esta dinámica, ya que han sido detectados procesos de dicotomización y otredad de este estilo en el discurso de Vox recogido por la prensa sobre la enmienda a la ley de cambio climático.

A nivel periodístico, la existencia de actores con discursos tan obstruccionistas como el analizado en este artículo dificulta aún más la ya complicada tarea (Boykoff, 2019; Mancinas, 2013) de comunicar la crisis climática. Así, llegados a este punto, sería interesante revisar el trabajo de Lakoff (2010), quien indica que existe una hipocognición respecto a los marcos que explican la crisis climática, es decir, hay carencia de argumentos y marcos para entender lo que 
está ocurriendo. Dada la responsabilidad periodística de comunicar eficazmente el problema climático al público de manera efectiva (Boykoff, 2019), cabe reflexionar aquí sobre hasta qué punto se debe prestar atención a los partidos políticos, grupos de interés y organizaciones cuyo objetivo es obstruir la acción climática, en lugar de a los actores que buscan soluciones acordes con la ciencia del clima y la situación de emergencia.

Esta investigación es una contribución empírica más a la línea de estudio sobre el contramovimiento climático, concretamente en el contexto político español. Futuros estudios pueden centrarse más en los discursos obstruccionistas de los partidos políticos sobre la acción climática y cómo se representan en la prensa, en otros medios de comunicación o incluso cómo se representan en los medios sociales. También sería interesante realizar estudios sobre la recepción de estos discursos, para ver si la polarización política en torno al cambio climático que retrata Vox se traslada también a la audiencia, o si ésta se alinea más con el consenso climático y rechaza los postulados del partido. En cualquier caso, la investigación sobre la obstrucción de la acción climática es ahora más necesaria que nunca, especialmente en el ámbito de la comunicación, que se ha convertido en un campo de batalla donde entran en juego múltiples intereses para influir en la opinión pública y en la toma de decisiones sobre cómo actuar para afrontar la crisis climática.

\section{NOTAS}

Este trabajo ha sido financiado por la Agencia Estatal de Investigación (AEI) y el Fondo Europeo de Desarrollo Regional (FEDER) (CSO2016-78421-R), y por el Ministerio de Ciencia, Innovación y Universidades (FPU18/04207).

\section{Referencias}

Aladro Vico, E., y Requeijo Rey, P. (2020). Discurso, estrategias e interacciones de Vox en su cuenta oficial de Instagram en las elecciones del 28-A. Derecha radical y redes sociales. Revista Latina de Comunicación Social, 77, 203-229. https://doi.org/10.4185/RLCS-2020-1455

Almiron, N., Boykoff, M., Narberhaus, M., y Heras, F. (2020). Dominant counter-frames in influential climate contrarian European think tanks. Climatic Change, 162(4), 2003-2020. https://doi.org/10.1007/s10584-020-02820-4

Black, R. (2018). Denied: The rise and fall of climate contrarianism. The Real Press.

Boykoff, M. (2019). Creative (Climate) Communications: Productive Pathways for Science, Policy and Society. Cambridge University Press. https://doi.org/10.1017/9781108164047

Boykoff, M., y Farrell, J. (2020). Climate change countermovement organizations and media attention in the United States. En N. Almiron y J. Xifra (Eds.), Climate Change Denial and Public Relations (pp. 121-139). Routledge.

Cárdenas-Rica, M. L., y Lozano González, A. A. (2020). El miedo como recurso persuasivo en el discurso político. En N. Ruiz-Alba y J. A. Moreno (Eds.), Debates contemporáneos sobre poder, política y medios de comunicación. Egregius.

Cook, J., Ellerton, P., y Kinkead, D. (2018). Deconstructing climate misinformation to identify reasoning errors. Environmental Research Letters, 13(2), 024018. https://doi.org/10.1088/17489326/aaa49f 
CSSN. (2021). The Structure of Obstruction: Understanding Opposition to Climate Change Action in the United States (CSSN Primer). Climate Social Sciences Network (CSSN). https://bit.ly/31X4v8D

Entman, R. M. (1993). Framing: Toward Clarification of a Fractured Paradigm. Journal of Communication, 43(4), 51-58. https://doi.org/10.1111/j.1460-2466.1993.tb01304.x

Fairclough, N., y Wodak, R. (1997). Critical Discourse Analysis. En T. A. Van Dijk (Ed.), Discourse as Social Interaction: Discourse Studies 2 (A Multidisciplinary Introduction) (pp. 258-284). Sage.

Fairclough, N. (2003). Analysing Discourse: Textual Analysis for Social Research. Routledge.

Farrell, J. (2016). Corporate funding and ideological polarization about climate change. Proceedings of the National Academy of Sciences, 113(1), 92-97. https://doi.org/10.1073/pnas.1509433112

Fernández-Reyes, R. (2014). Infoxicación en la comunicación del cambio climático. Mitigación y adaptación. Ámbitos. Revista Internacional de Comunicación, 26, 66-76. https://doi.org/10.12795/ambitos.2014.i26.07

Ferreira, C. (2019). Vox como representante de la derecha radical en España: Un estudio sobre su ideología. Revista Española de Ciencia Política, 51, 73-98. https://doi.org/10.21308/recp.51.03

Fisher, D. R., Waggle, J., y Leifeld, P. (2013). Where does political polarization come from? Locating polarization within the U.S. climate change debate. American Behavioral Scientist, 57(1), 70-92. https://doi.org/10.1177/0002764212463360

Forchtner, B. (Ed.). (2019). The far right and the environment: Politics, discourse and communication. Routledge. https://doi.org/10.4324/9781351104043

Forchtner, B., y Lubarda, B. (2021, 1 de noviembre). The far right has moved from climate denial to obstructing climate action. Open Democracy. https://bit.ly/3dP8dna

Forchtner, B., Kroneder, A., y Wetzel, D. (2018). Being skeptical? Exploring far-right climate-change communication in Germany. Environmental Communication, 12(5), 589-604. https://doi.org/10.1080/17524032.2018.1470546

González, M. (2021, 11 de septiembre). Vox alimenta el negacionismo para pescar votos entre los perdedores de la transición energética. EL País. https://bit.ly/30ockDs

Gould, R. (2019). Vox España and Alternative für Deutschland: Propagating the crisis of national identity. Genealogy, 3(4), 64. https://doi.org/10.3390/genealogy3040064

Hultman, M., Björk, A., y Viinikka, T. (2019). The far right and climate change denial. Denouncing environmental challenges via anti-establishment rhetoric, marketing of doubts, industrial/breadwinner masculinities enactments and ethno-nationalism. En B. Forchtner (Ed.), The Far Right and the Environment: Politics, Discourse and Communication. Routledge.

IPCC. (2021). Climate Change 2021: The physical science basis. Contribution of Working Group I to the Sixth Assessment Report of the Intergovernmental Panel on Climate Change. IPCC. https://www.ipcc.ch/report/ar6/wg1/

Jylhä, K. M., y Hellmer, K. (2020). Right-wing populism and climate change denial: The roles of exclusionary and anti-egalitarian preferences, conservative ideology, and antiestablishment attitudes. Analyses of Social Issues and Public Policy, 20(1), 315-335. https://doi.org/10.1111/asap.12203

Lakoff, G. (2010). Why it matters how we frame the environment. Environmental Communication, 4(1), 70-81. https://doi.org/10.1080/17524030903529749 
Lockwood, M. (2018). Right-wing populism and the climate change agenda: Exploring the linkages. Environmental Politics, 27(4), 712-732. https://doi.org/10.1080/09644016.2018.1458411

Machin, D., y Mayr, A. (2012). How to do critical discourse analysis: A multimodal introduction. SAGE.

Mancinas-Chávez, R. (2013). El silencio mediático. Reflexión en torno a las razones de los medios de comunicación para no hablar del cambio climático. En R. Mancinas Chávez (Ed.), Medios de comunicación y cambio climático (pp. 233-248). Universidad de Sevilla. https://idus.us.es/xmlui/handle/11441/30565

Martín-Sosa, S. (2021). Apuntes metodológicos para el estudio del negacionismo climático en los medios escritos. Communication \& Methods, 3(1), 56-66. https://doi.org/10.35951/v3i1.111

McCright, A. M., y Dunlap, R. E. (2010). Anti-reflexivity. The American conservative movement's success in undermining climate science and policy. Theory, Culture \& Society, 27(2-3), 100-133. https://doi.org/10.1177/0263276409356001

McKie, R. E. (2019). Climate change counter movement neutralization techniques: A typology to examine the climate change counter movement. Sociological Inquiry, 89(2), 288-316. https://doi.org/10.1111/soin.12246

Moreno, J. A., y Almiron, N. (2021). Representación en la prensa española del papel de la agricultura animal en la crisis climática. Estudios Sobre El Mensaje Periodístico, 27(1), 349-364. https://doi.org/10.5209/esmp.73745

Moreno, J. A., y Ruiz-Alba, N. (2021). ¿Periodismo o greenwashing? Patrocinadores de la COP25 ChileMadrid en la prensa española. Revista Mediterránea de Comunicación, 12(2), 285-300. https://www.doi.org/10.14198/MEDCOM.19089

Parratt Fernández, S., Mera Fernández, M., y Carrasco Polaino, R. (2020). La relevancia del cambio climático en la prensa española: Análisis comparativo de El País, El Mundo y ABC. OBETS. Revista de Ciencias Sociales, 15(2), 625. https://doi.org/10.14198/OBETS2020.15.2.09

Piltz, R. (2008). The Denial Machine. Index on Censorship, 37(4), 72-81. https://doi.org/10.1080/03064220802561366

Rama, J., Zanotti, L., Turnbull-Dugarte, S. J., y Santana, A. (2021). VOX: The rise of the Spanish populist radical right. Routledge. https://doi.org/10.4324/9781003049227

Reisigl, M., y Wodak, R. (2001). Discourse and discrimination. Rhetorics of racism and antisemitism. Routledge.

Robaina, E. (2019, 5 de junio). VOX y su argumentario contra el cambio climático: 'No vamos a malgastar más dinero en esta estafa'. La Marea. https://bit.ly/3m4EPOv

Schaller, S., y Carius, A. (2019). Convenient Truths. Mapping climate agendas of right-wing populist parties in Europe. Adelphi. https://bit.ly/33iZXto

Turnbull-Dugarte, S. J. (2019). Explaining the end of Spanish exceptionalism and electoral support for Vox. Research \& Politics, 6(2), 205316801985168. https://doi.org/10.1177/2053168019851680

van der Linden, S., Leiserowitz, A., Rosenthal, S., \& Maibach, E. (2017). Inoculating the public against misinformation about climate change. Global Challenges, 1(2), 1600008. https://doi.org/10.1002/gch2.201600008 
Vowles, K., y Hultman, M. (2021). Scare-quoting climate: The rapid rise of climate denial in the Swedish far-right media ecosystem. Nordic Journal of Media Studies, 3(1), 79-95. https://doi.org/10.2478/njms-2021-0005

Wodak, R., y Meyer, M. (2009). Methods of critical discourse analysis (2nd ed.). Sage.

\section{Semblanza de los autores}

Jose A. Moreno es investigador predoctoral en el programa de doctorado en Comunicación de la Universitat Pompeu Fabra de Barcelona, dentro del Programa de Formación del Profesorado Universitario (FPU) del Ministerio de Ciencia, Innovación y Universidades. Miembro del grupo de investigación Critical Communication (CRITICC) y del proyecto de investigación THINKClima (CSO2016-78421-R) en el Departamento de Comunicación de la Universitat Pompeu Fabra. Su línea de investigación se centra en la comunicación del cambio climático y los grupos de interés.

Gina Thornton es una investigadora independiente, con experiencia como asistente de investigación en el grupo de investigación UNICA de la Universitat Pompeu Fabra, que actualmente trabaja como técnica de proyectos en la Agencia de Gestión de Ayudas Universitarias y de Investigación (AGAUR). Participó en el Seminario Avanzado de Estudios del Discurso en 2020 (SAED) y su línea de investigación se centra en la comunicación política, los estudios de extrema derecha y el análisis crítico del discurso. 